\title{
Research on Dynamic Reactive Power Optimization of Distribution Network Based on Modern Mathematical Algorithms
}

\author{
Lihong Bai ${ }^{1, a}$ \\ ${ }^{1}$ Department of Basic courses, Gansu Institute of Architectural Technology, Lanzhou, China \\ aBailihong0305@126.com
}

Keywords: Distribution Network, Dynamic Reactive Power Optimization, Mathematical Algorithms

\begin{abstract}
With the development of science and technology, there has been growing the grid size, the growing demand for electricity and the extent of increasing the electricity market. In this paper, a mathematical morphology and genetic algorithm based dynamic reactive power optimization method for distribution network is proposed. By means of constructing mathematical morphology filter, the binary image composed by allelic genes of chromosome is filtered and the problem that deals with the action times of voltage regulating devices for compensation is turned into filtering problem of discrete binary image. With further automate scheduling, distribution automation and unmanned substation reactive power optimization of distribution line running made an urgent request. Reactive power optimization can improve the voltage passing rate, increasing the reliability of the system, the power quality, and the system runs the security and economy of the perfect combination, very broad application prospects.
\end{abstract}

\section{Theoretical Introduction of Dynamic Reactive Power Optimization}

Reactive power optimization of power system dynamic network structure refers to the parameters of the system, the next day under each load bus of active and reactive and active power output curve for a given situation, and by adjusting the reactive power generator reactive power compensation equipment and load tap, and meet the constraint conditions, solving the optimal reactive power switching device control policy makes the whole day minimum loss of power grid.

Optimization of power system operation, in order to track changes in the load line, to keep the system is always in the best state, in theory requires constant reactive power optimization. However, in actual operation system, due to the application and operating difficulties, and not always reactive power optimization, because of frequent non-reactive power optimization will not only cause the device switching too often influence the practical life of the equipment, but also increase the operating personnel work intensity. Dynamic reactive power optimization by adjusting the generator reactive power, output capacitors and load tap, and meet a variety of operating conditions constrained minimum power loss across the grid ${ }^{[1]}$.

Dynamic reactive power optimization is active in the network structure and parameters of each load bus the next day, the next reactive and active power output curve of a given case, by adjusting the generator reactive power, output capacitor bank and load transfer tap voltage transformer, to meet a variety of operating constraints conditions power loss across the grid is minimized. Distribution and transmission networks compared with the two distinct features: 1) low voltage levels; 2) weak ring or radial run. Meanwhile, the distribution network involved in reactive power optimization of control equipment and the grid is also somewhat different: the main transformer to adjust joints; capacitor switching, including centralized installation in medium voltage bus and capacitors installed on the dispersion medium voltage lines; contact switch switching, network reconfiguration, etc. that is commonly referred to. Therefore, the distribution network reactive power optimization can target their own characteristics, make improvements in the algorithm. Common practice is the best compromise in whichever computational efficiency and global, that is optimized to obtain a good result on the basis of a simplified model. 


\section{Modern Mathematical Algorithm}

Dynamic reactive power optimization goal is qualified under the premise that the voltage to minimize system power loss, and the number of operations to meet the compensation requirements regulating equipment. Usually net loss of 24 day period and a minimum, each time the voltage is not limited to, the number of operations to compensate regulating equipment meets the requirements for the constraints. To reduce the operating times of the compensation regulator device, on the one hand be compensated as long as possible to ensure a time regulator device does not operate, on the other hand is considered the compensation regulator device operation continuity. Select structure element shown in Fig. 1, wherein the B1-B9 structural elements: B1 and B: represents the period of two adjacent transformers (or capacitor) range of motion for a position ((1 group) ${ }^{[2]}$. Fig.1 shows the selection of structure element.

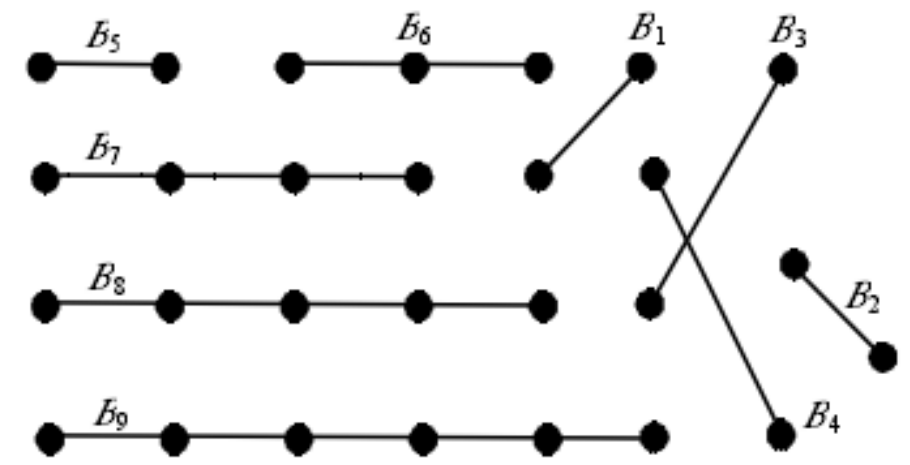

Fig. 1.The selection of structure element

Dynamic Reactive Power Optimization for the current method of calculating capacity, the number of operations to compensate the regulator device constraints difficult to handle such issues, this paper draw on the idea of mathematical morphology, structure of mathematical morphology filters on chromosome allele from each period of a collection of binary image filtering, improved genetic algorithm simple crossover and mutation operations. The genetic algorithm is optimal method of a natural process of evolution of the search process through simulation. Genetic algorithms and other evolutionary algorithms such as evolutionary programming, evolutionary strategy, and genetic programming classification system based on mathematical modeling of the same evolutionary process and the specific implementation, but it also has its own unique ideas.

Genetic algorithms are essentially strings op mode operation. Thus, by analyzing the patterns of genetic variation in the operation, you can understand what the nature of being a continuation of nature is discarded, thereby grasp the essence of the genetic algorithm. Visible genetic algorithm in handling this mode, it is also implicit in many of the higher processing mode, that is, the genetic algorithm when dealing with a limited number of individuals deal with a lot of models and the information they carry. This recessive genetic algorithm for parallel processing mechanism is an important reason for the effectiveness ${ }^{[3]}$.

\section{The Dynamic Reactive Power Optimization Based on Genetic Algorithm}

Number of actual system commissioning group capacitor banks are integers, the paper takes a capacitor bank and put into operation the number of groups for the control variables, using integer encoding control variable encoding format is divided into two parts, one for the number of groups and put into operation, part of to put into operation time. With this two-stage encoding approach has two advantages: (1) to shorten the length of chromosome to $M$ sets of the capacitor bank, the maximum allowable number of operations $\mathrm{K}$, for example, two-stage coding chromosome length $2 \mathrm{KM}$, and to the capacitor bank at each time point capacity of chromosome encoding, its length is 24M.(2) Eliminate infeasible code ${ }^{[4]}$.

Genetic algorithms work steps are: 1) the parameters of the problem, select the appropriate coding strategies, such as the length of the string on behalf of the individual machines, such as its composition. 2) The part individual of the initial population is randomly selected. 3) Select the 
appropriate fitness function, which is calculated for each individual's fitness function. 4) According to genetic strategy, select the number of population and determine the selection, crossover and mutation of three methods and crossover probability and mutation probability. 5) Strategy based on genetic algorithms, continuous use of selection, crossover and mutation three kinds of genetic operators to act on the initial population, allowed the formation of groups of offspring generations, until an optimal individual so far. 6) The best individual fitness function is most optimal output.

Using competitive merit-based crossover operator, first produced four offspring from the parent, and then chose the best fitness value of two chromosomes in four chromosomes as the parent crossover operator progeny. The operator can try to ensure that the individual has an excellent pattern being destroyed by hybridization operation, and can increase the degree of dispersion of the population to generate a new search space. Conditions by the end of the algorithm composed of two aspects: (1) when the number of iterations is greater than the specified maximum number of iterations to stop the iteration, (2) optimal solution in successive iterations $\mathrm{k}$ remains unchanged, stop iteration, $\mathrm{k}$ depends on the size scale of the problem and difficulty may be. Fig.2 shows the flow chart of dynamic; reactive power optimization by genetic algorithm.

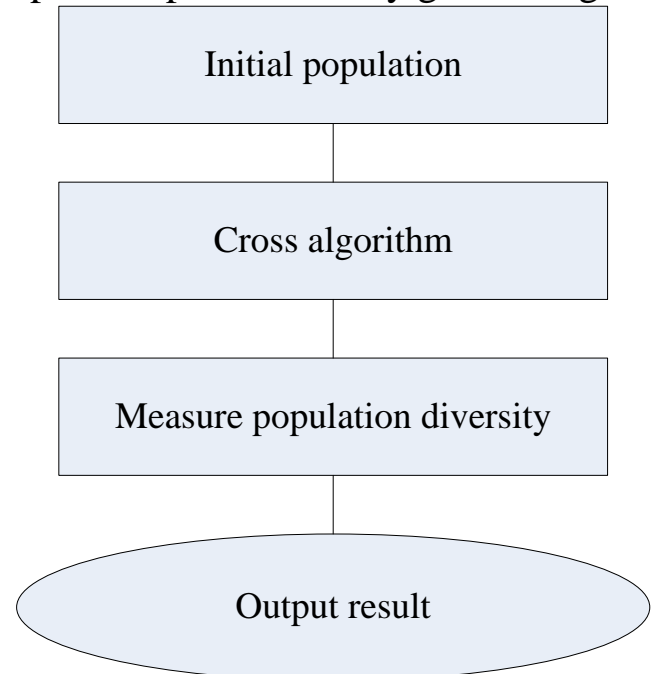

Fig. 2.The flow chart of dynamic; reactive power optimization by genetic algorithm

\section{The Result Analysis}

There is calculation and analysis in a region 40 bus distribution system as an example. The power compensation equipment, including 14 sets of regulator load tap changer ((T1 Tl4, stalls from 1 to 17 ) and 7 of reactive power compensation point (C $1 \sim \mathrm{C} 7$ ). Set the system voltage range of 0.95 to allow $1.07 \mathrm{pu}$, power reference value of 100MVA, distribution network of dynamic reactive power optimization before, the system network loss is $34.63 \mathrm{MW}$. Using VB language, the CPU oscillator frequency is $1.5 \mathrm{GHz}, 256 \mathrm{M}$ memory for the computer programming network parameters and load data provided by the regional dispatch center ${ }^{[5]}$.

Filtering by selecting the appropriate structural elements, we can limit the number of operations to compensate the regulator device, reducing the distribution network of dynamic reactive power optimization complexity. Based crossover and mutation genetic traits, reducing the possibility of excellent Majesty destroyed like better than a simple ordinary mathematical algorithms optimization results, calculate faster. Lower dynamic optimization methods Loss decreased amount, net loss decreased rate of $14.99 \%$, but the number of operations and lower compensation regulating equipment, reactive power optimization effect is ideal.

The use of dynamic optimization methods SGA, IGA and article after load tap T: numbers of operations were 16, 14 and 80 of the switching capacitors C1 curve in Figure 3. Figure 3 shows, as compared with the static optimization, dynamic optimization method can significantly reduce the number of operations of the parallel capacitor C1. The figure below are described in this paper the dynamic optimization method can significantly reduce the number of operations to compensate the 
regulator device, to avoid frequent changes in transformer stalls, can ensure economic operation of the distribution system, but also reduce the probability of equipment failure compensation regulator. Fig.3 shows the Curves of on-load transformer T3 tap changer.

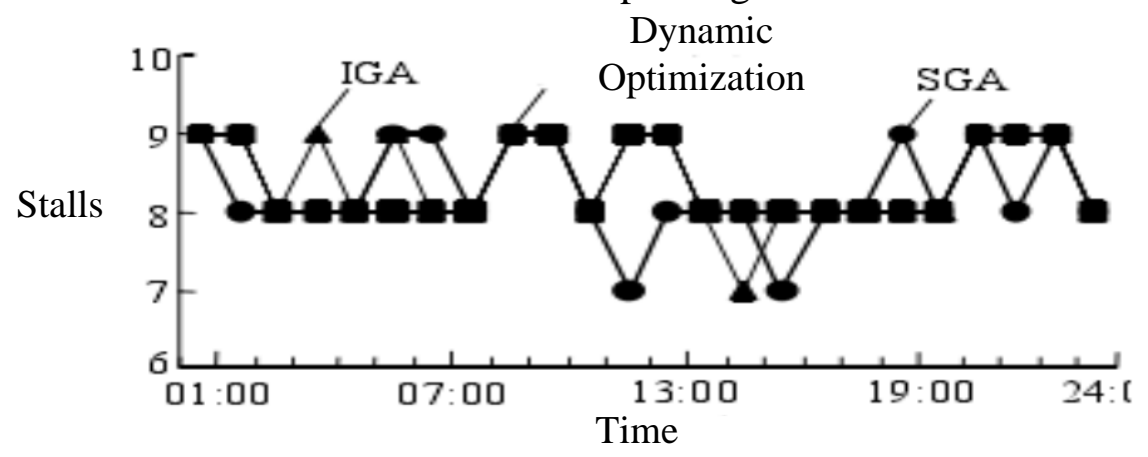

Fig. 3.The Curves of on-load transformer T3 tap changer

\section{Conclusions}

Reactive power optimization of power system control is to ensure safety, an effective means of economic operation, is an important measure to reduce network active power loss and improve the voltage quality. Research therefore reactive power optimization problem with both theoretical significance, but also has practical value. Reactive Power Optimization difficulty is to establish the validity of the model and optimization methods. The mathematical algorithm based on the characteristics of the natural immune response, the clonal selection, clonal expansion, not stimulate cell demise and the generation of memory cells, immune and other immune mechanisms introduced to supplement the basic genetic algorithm, the algorithm was improvements. The result based on the proposed method is compared with that based on the static optimization and other algorithms on the same system, great success in comparison the constraints of operation times and the total of profit, and this has shown correctness and effectiveness of the proposed method.

\section{Acknowledgment}

2014 Scientific Research Projects in Tertiary Institutions, Gansu Province, Project Number: 2014A-142.

\section{References}

[1] Suresh, R, Kumarappan, N. Genetic algorithm based reactive power optimization under deregulation Information and Communication Technology in Electrical Sciences (ICTES 2007), 2007. ICTES. IET-UK International Conference on, 20-22 Dec. 2007: 150-155.

[2] Biao Pang Wei Long, Lin Hu .Development \& Application of Reactive Power Optimization Software for Regional Power Network Intelligent Human-Machine Systems and Cybernetics, 2009. IHMSC '09. International Conference on, 26-27Aug. 2009: 376-379.

[3] Xin Ma Rui-Lan Liu Reactive power optimization in power system based on improved niche genetic algorithm Computer Design and Applications (ICCDA), 2010 International Conference on 25-27 June 2010: V3-413-V3-416.

[4] Zhou Shan Liu Wen-zhou, Cai Chang-qing, Zhang Li. Reactive power optimization of distribution network based on GA with simulated annealing selection Computer Science \& Education (ICCSE), 2011 6th International Conference on, 3-5 Aug. 2011: 1054-1057.

[5] Sattianadan, D of capacitor in Sudhkaran, M, Vijayakumar, K., Vidyasagar, S. Optimal placement radial distribution system using PSO Sustainable Energy and Intelligent Systems (SEISCON 2011), International Conference on, July 2011: 326-331. 